\title{
ENGINEERING AND DESIGN OF A CO2 PHASE CONTRAST INTERFEROMETER SYSTEM FOR DIII-D
}

\author{
by \\ R.D. PHELPS and S. CODA*
}

*Massachusetts Institute of Technology

Prepared under

Contract No. DE-AC03-89ER51114

for the U.S. Department of Energy

\section{GENERAL ATOMICS PROJECT 3466}

NOVEMBER 1994

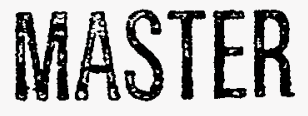




\section{DISCLAIMER}

This report was prepared as an account of work sponsored by an agency of the United States Government. Neither the United States Government nor any agency thereof, nor any of their employees, make any warranty, express or implied, or assumes any legal liability or responsibility for the accuracy, completeness, or usefulness of any information, apparatus, product, or process disclosed, or represents that its use would not infringe privately owned rights. Reference herein to any specific commercial product, process, or service by trade name, trademark, manufacturer, or otherwise does not necessarily constitute or imply its endorsement, recommendation, or favoring by the United States Government or any agency thereof. The views and opinions of authors expressed herein do not necessarily state or reflect those of the United States Government or any agency thereof. 


\section{DISCLAIMER}

Portions of this document may be illegible in electronic image products. Images are produced from the best available original document. 


\section{INTRODUCTION}

This report describes the development of a $\mathrm{CO}_{2}$ laser interferometer system, the engineering, design and installation of the hardware, and the selection of materials specific to the requirements of a $\mathrm{CO}_{2}$ laser diagnostic. A brief description of system operation is included.

A phase contrast interferometer diagnostic has been designed and installed on the DIII-D tokamak to enhance studies of the physical characteristics of plasma turbulence, and specifically to analyze plasma density fluctuations in the boundary region of the plasma. A 20 watt $\mathrm{CO}_{2}$ laser beam, operating at the 10.6 micron wavelength, is expanded to a diameter of $76 \mathrm{~mm}$ and directed through a series of mirrors which provide for entry of the beam into the vessel at a point $70 \mathrm{~cm}$ above the midplane at the 285 degree toroidal location. After being reflected from a mirror inside the vessel, the beam is directed downward so that it passes through the edge of the plasma immediately in front of a fourstrap fast wave current drive RF antenna. The laser beam is then reflected by a second internal mirror and exits the vessel $70 \mathrm{~cm}$ below the midplane (also at 285 degrees) returning to an optical table through a final series of external steering mirrors. Figure 1 shows the beam path relative to the vessel wall and ports; Fig. 2 is an internal vessel photo showing the entrance and exit ports for the beam (the shutters are closed) and their relationship to the antenna.

Upon traversing the plasma edge inside the vessel, the $\mathrm{CO}_{2}$ laser beam is unchanged in amplitude. However, the beam undergoes a phase shift proportional to the vertical line integral of the fluctuating component of the plasma density along the beam path. The phase contrast technique, in combination with an imaging configuration, is then used to convert the resulting phase shift information into an amplitude while creating an image of the plasma edge on an array of 16 detectors. Hence, each of the 16 detector elements corresponds to a specific radial location in the plasma and generates a signal proportional to the line-integrated density perturbation at that location.

The $\mathrm{CO}_{2}$ laser source, the initial and final beam steering mirrors, the detectors, and the associated electronics are all located on a physically enclosed optical table. 


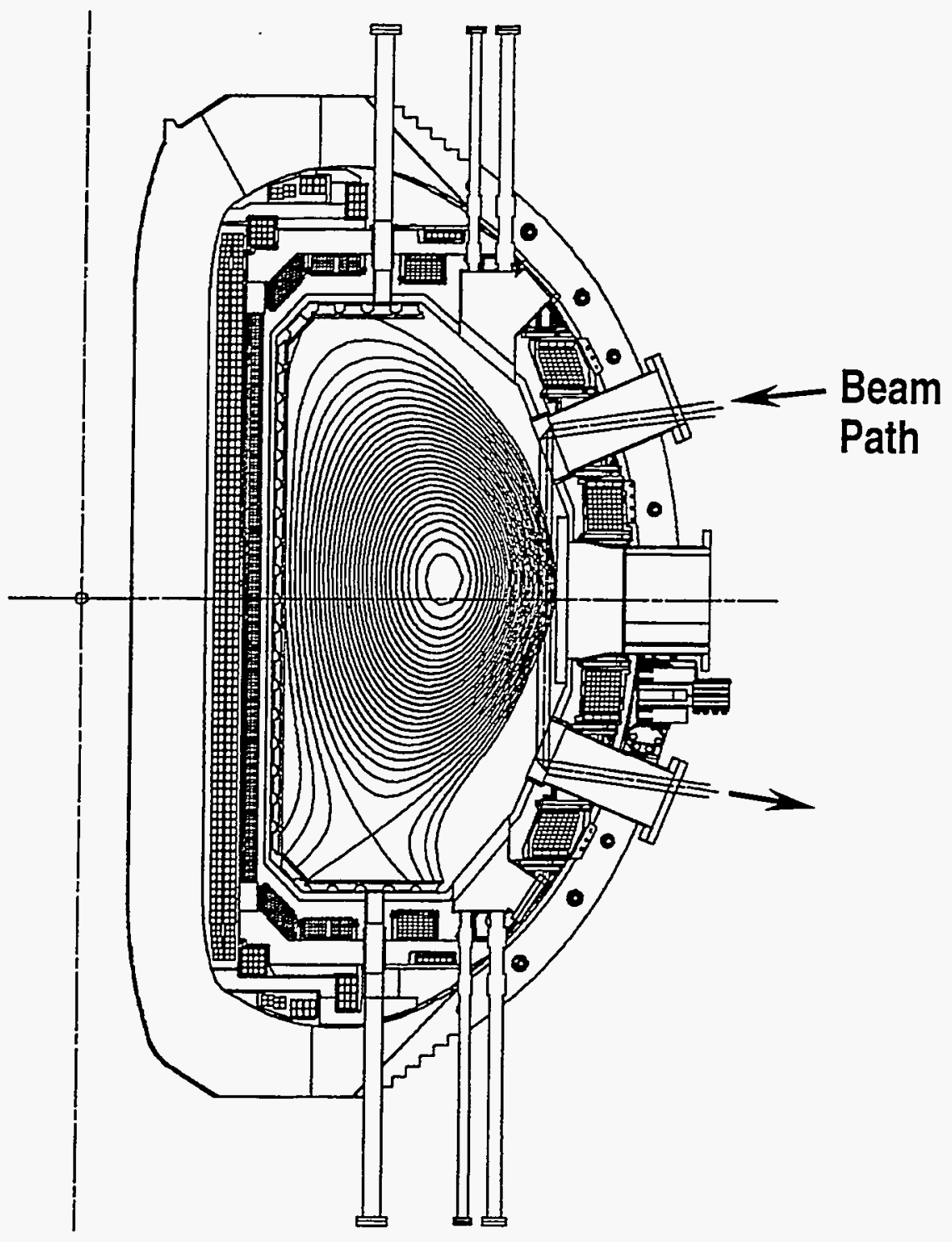

Fig. 1. DIII-D elevation at $285^{\circ}$ phase contrast interferometer diagnostic. 


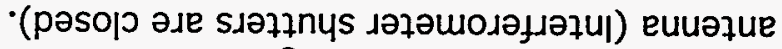

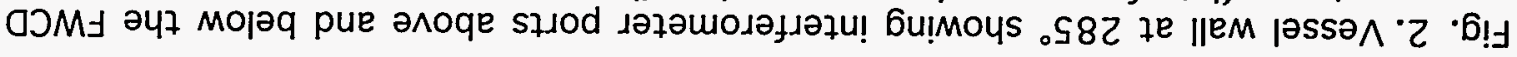

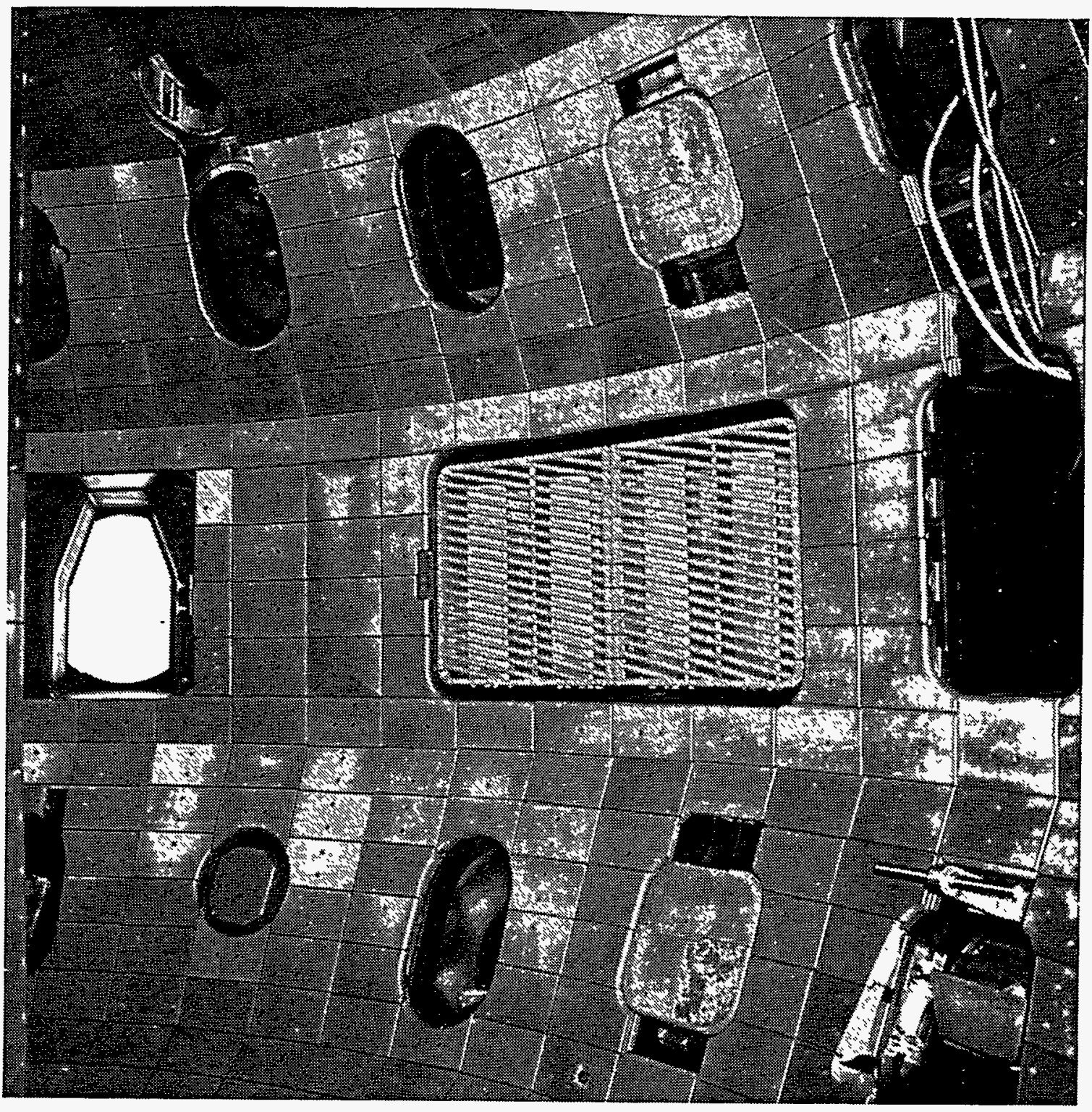




\section{SYSTEM DESIGN}

The $\mathrm{CO}_{2}$ laser beam is generated by equipment mounted on a vertically oriented $4^{\prime} \times 6$ ' optical table west of the DIII-D vessel on the pit floor. The $3.7 \mathrm{~mm}$ diameter source beam is first expanded to $76 \mathrm{~mm}$ using a lens and an off-axis parabolic mirror, and then transmitted into the vessel through a series of large diameter $(229 \mathrm{~mm})$ precision adjustable mirrors. Smaller diameter adjustable mirrors are used in the ports through which the beam passes into and out of the vessel. The return beam also passes through a series of mirrors external to the vessel, plus a beam contracting parabolic mirror on the optical table before being directed to the analytical instruments. The $\mathrm{CO}_{2}$ laser beam is everywhere enclosed to protect the equipment and to minimize inadvertent personnel exposure. The overall laser beam path is shown pictorially in Fig. 3.

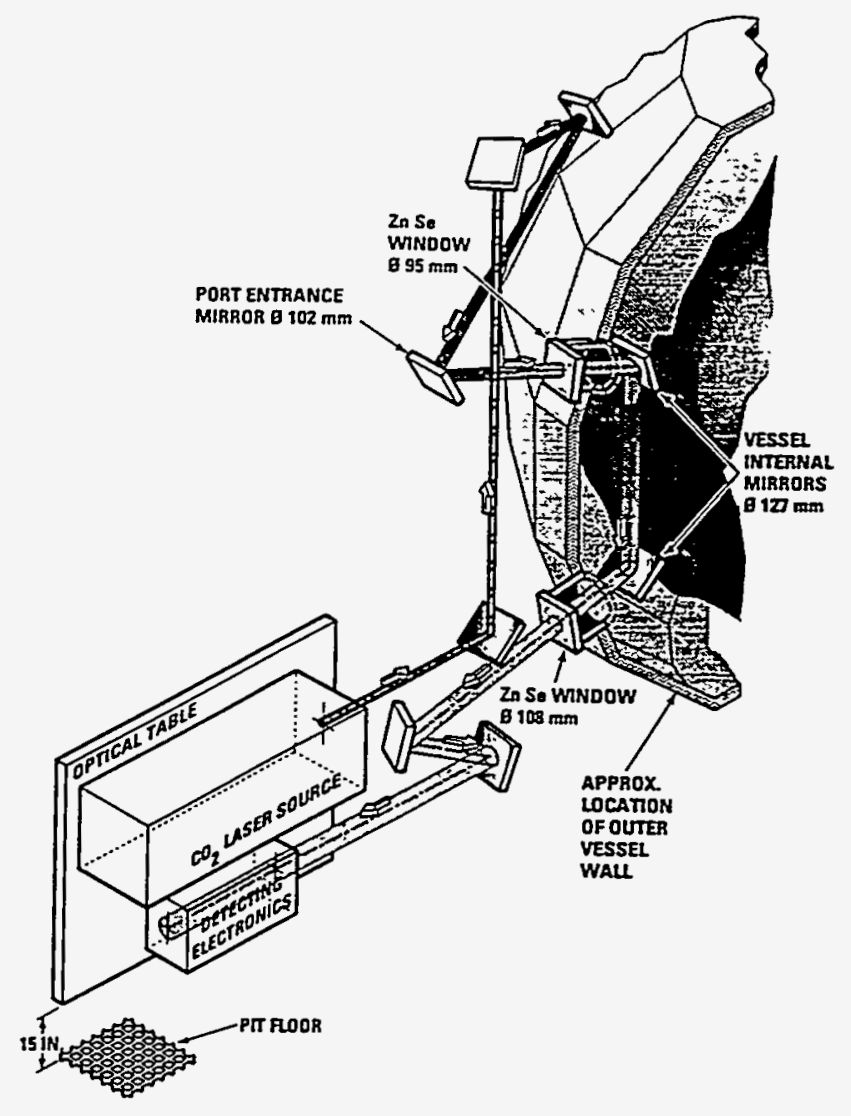

Fig. 3. $\mathrm{CO}_{2}$ laser beam path. 
The port windows through which the beam passes into and out of the vessel must be transparent primarily to the 10.6 micron wavelength, but must also transmit sufficient visible light to allow for beam alignment procedures. The window material must also possess sufficient strength to withstand the pressure differential of a full vacuum. The material chosen for this application is zinc selenide, $9.5 \mathrm{~mm}$ thick, at a diameter of $95 \mathrm{~mm}$ for the entrance window, and $108 \mathrm{~mm}$ for the exit window. The window surfaces are coated with a thorium fluoride anti-reflective material to enhance transmission. The plane of the port windows is positioned approximately one degree off-normal from the port axis when installed on the vessel port extensions. This slight deviation is intended to minimize interference effects on the primary laser beam due to reflections at the window interface.

The vessel internal mirrors are $127 \mathrm{~mm}$ in diameter and are fabricated from fused quartz with an aluminum reflecting surface 0.1 microns thick. The reflecting surface is further protected by a layer of silicon monoxide 0.15 microns thick. The mirrors are located in a mounting ring which is in turn attached to a support assembly. This arrangement provides for biaxial adjustment of the mirror. However, only the poloidal axis remains fully movable; the toroidal axis, once adjusted, remains fixed. The fully adjustable poloidal axis of the mirror can be manipulated from outside the vessel by way of a linear mechanical vacuum feedthrough containing an integral micrometer.

Each of the internal mirrors is protected by an inconel shield and shutter mechanism. The shutter is attached to an inconel shield plate which is bolted to the inside vessel wall at the port interface [1].

Each of the six individual external mirrors is housed in a precision Aerotech mount equipped with built-in fine adjustments for the two plane axes of the mirror. This adjustment allows for a positional resolution of 0.04 arc-seconds or better per seven degrees of mirror movement. Gross adjustment of these mirrors is accomplished by installing the precision mirror mount on a gimbaled support mechanism having two degrees of freedom. The gimbal supports are fixed in position once the initial system alignment has been completed, and final adjustments to obtain precise alignment can then be made at the Aerotech mirror mount itself.

Five of the six external mirrors are fabricated of Pyrex glass to provide a "clear" aperture diameter of $206 \mathrm{~mm}$. The sixth mirror, is located just in front of the entrance 
window to the vessel. All of the external mirror surfaces are coated with a layer of aluminum to enhance reflectivity, and the aluminum is in turn protected by a layer of silicon monoxide. 


\section{LASER SOURCES}

The primary laser source for the interferometer system is a self-contained carbon dioxide laser operating at the 10.6 micron (infrared) wavelength with an output power of 20 watts. A high degree of stability ( $0.5 \%$ in power and $300 \mathrm{kHz}$ in frequency) is achieved through feedback control of the cavity length. The position of the output coupler, which is mounted on a piezo-electric translator, is adjusted in real time for maximum power. The output laser beam is gaussian with a diameter of $3.7 \mathrm{~mm}$ at the source and a divergence of 3.6 milliradians. A continuously variable $\mathrm{ZnSe}$ attenuator is used to reduce the laser power to a level which precludes detector saturation. The source beam is folded several times on the optical table to allow a free expansion along an optical path of approximately 1.6 meters. The beam is then passed through an $8 x$ telescope consisting of a $\mathrm{ZnSe}$ lens and an off-axis parabolic mirror. The final result is a collimated gaussian beam $76 \mathrm{~mm}$ wide.

A secondary laser source for this interferometer system is produced by a small helium-neon laser operating at the 632.8 nanometer wavelength at a power of 16 milliwatts. This laser is used for alignment purposes. Rather than operate the $\mathrm{CO}_{2}$ laser during alignment, the He-Ne laser is used. This choice stems from the visibility of the small diameter He-Ne laser as opposed to the larger $\mathrm{CO}_{2}$ laser which is invisible to the human eye as well as being a personnel hazard. 


\section{VESSEL PORT FLANGE}

The vessel port is constructed with a standard 17 -in. diameter vacuum flange to which the interferometer port flange is attached. The port flange includes the window mounting flange assembly and associated mirror support tube, plus a pair of small vacuum flange stubs for mounting the mirror adjustment feedthrough actuator and the shutter actuator. The window mounting flange is positioned with a one degree axial offset to obtain a prescribed amount of misalignment intended to eliminate interferences in the system due to reflection of the laser beam from the window back along the incident axis. 


\section{PORT WINDOWS}

The zinc selenide windows installed on the vessel are supplied by II-VI, Inc. The entrance window is $95 \mathrm{~mm}$ in diameter and the exit window is $108 \mathrm{~mm}$. Both windows are $9.5 \mathrm{~mm}$ thick. A series of O-rings has been designed into the window support flange to obtain a high vacuum seal between the window material surface and the flange. This arrangement places a minimum of stress on the $\mathrm{ZnSe}$ material while providing good flexibility at the contact surfaces. To monitor any possible leaks through the primary Oring seal, a small pumpout and leak check access hole was machined into the window support flange between the first and second O-ring grooves. This access can be used for leak checking, and if the O-ring seal is determined to be leaking, the pumpout can be connected into the tokamak secondary vacuum system to minimize the pressure difference across the leaking primary seal O-ring.

The window flange is bolted to a vacuum flange welded to a short section of pipe which is in turn welded into the port flange which bolts to the vessel. The pipe provides physical support for the window assembly and mirror mount tube. A series of six radially oriented bolts at the end of the pipe are tightened against the mirror mount tube thus fixing its position in the pipe and providing physical support to minimize any effect of the long lever arm between the window mounting flange and the mirror mounting point at the opposite end of the tube. 


\section{INTERNAL MIRROR MOUNTS}

The fused quartz mirrors located inside the vessel are contained in a mounting ring designed for routine adjustment around the horizontal axis only. A toroidal adjustment is designed into the assembly, but can be manipulated only at the time of initial assembly of the parts in the vessel. Once the toroidal position of the mirror has been set, the toroidal movement of the mirror mount is fixed by welding.

The mirror mounting ring is affixed to the mirror support assembly by a pair of precision ground pivot pins which pass through graphite bearings enclosed in adjustable retainers. The graphite bearings (POCO AXF-5Q) are precision ground to obtain a minimum clearance fit with both the pivot pins and the inside diameter of the retainers. This is intended to prevent any undesirable movement between these parts which might affect the accuracy of adjustment or operation. The bearing retainer is threaded into the support assembly to allow for precise adjustment of friction between the bearing and its supports. Once the assembly of the bearing is complete, and adjustments are made, the bearing housing is spot welded to prevent any subsequent loosening. Figure 4 shows the general arrangement of the mirror mount installed in the 285 degree $\mathrm{R}+1$ port. 


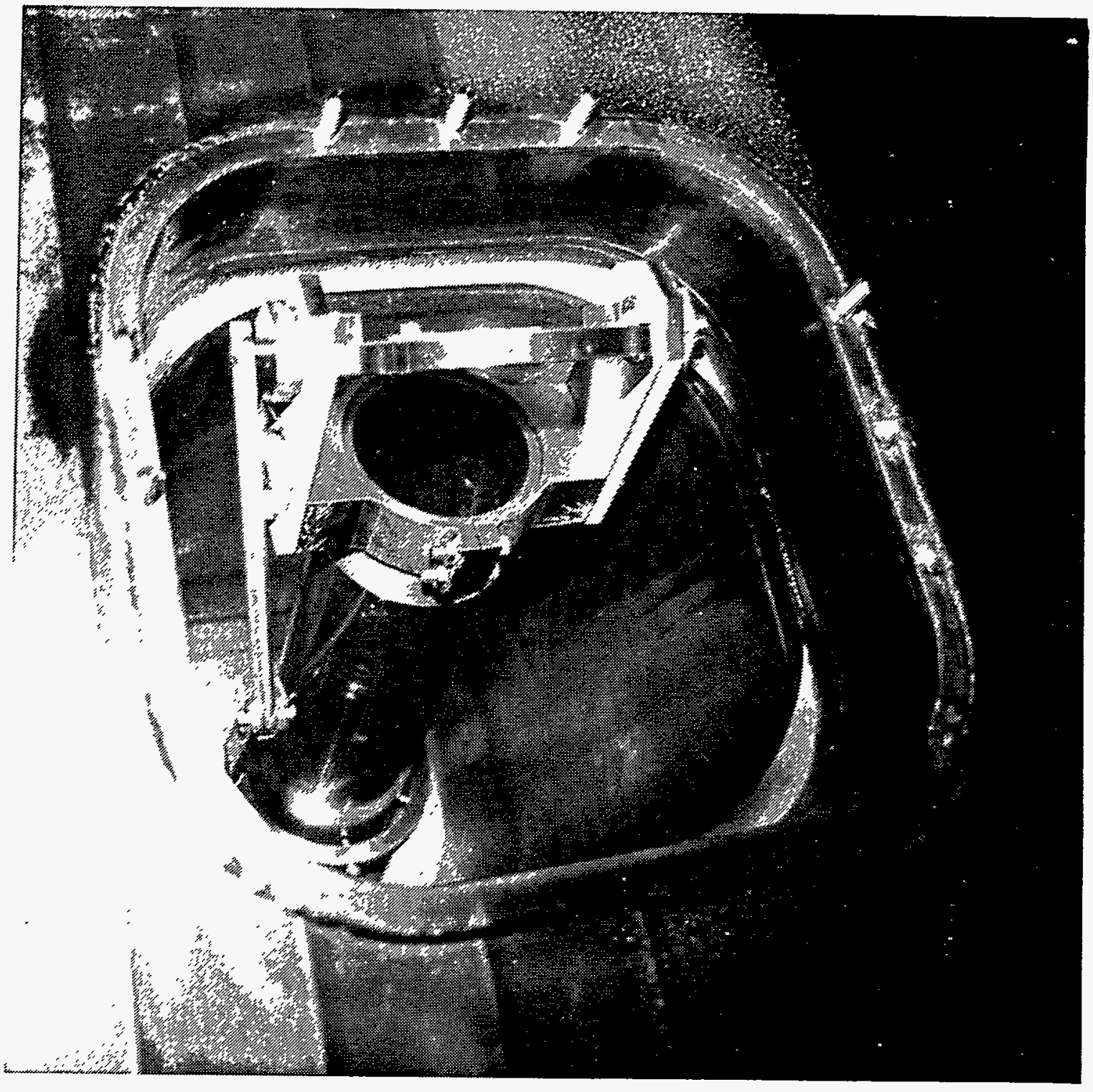

Fig. 4. Internal mirror mount installation- $R+1285^{\circ}$. 


\section{MIRROR ADJUSTMENT AND SHUTTER DRIVE}

The shutter drive consists of a linear mechanical vacuum feedthrough, equipped with a small air cylinder, by which the operating push rod is moved through a short distance to accomplish the opening and closing movements of the shutter (see Fig. 5). The feedthrough is mounted on the port closure flange attached to the vessel. To prevent electromagnetically induced loads in the push rod assembly, an electrical break is formed by installing a small section of ceramic material between the actuator and the end of the push rod. Admission of air to the drive cylinder is controlled by the vessel operating logic system which allows the shutter to be opened or closed only under prescribed program conditions.

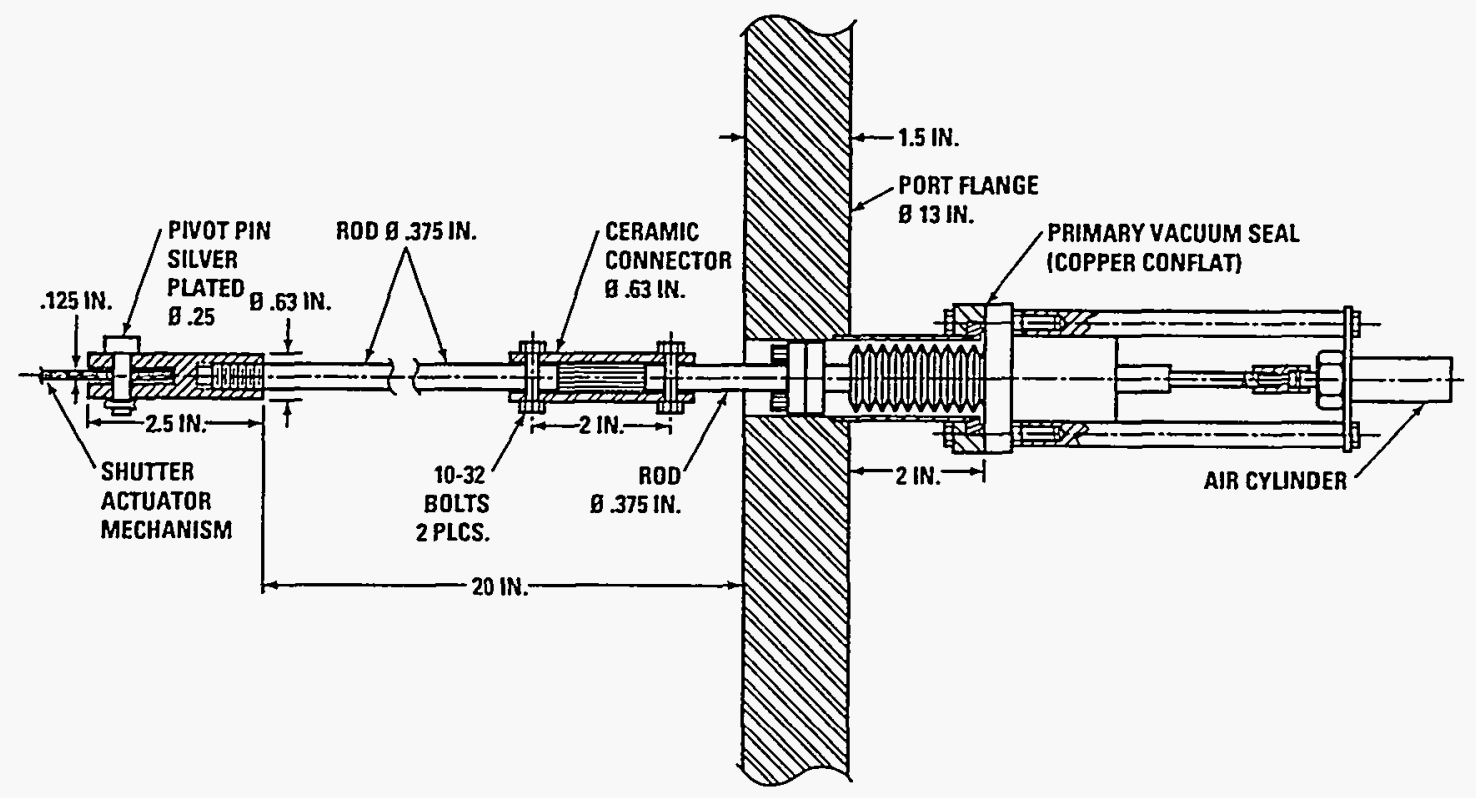

Fig. 5. Shutter actuator mechanism.

The final internal mirror adjustment is accomplished with a micrometer equipped linear feedthrough by which precise actuator movements of less than $0.025 \mathrm{~mm}$ can be made. As with the shutter push rod, a small section of ceramic material is inserted between the micrometer feedthrough actuator and the mirror adjustment push rod to prevent development of undesirable electromagnetic forces in the actuator hardware.

The mirror mount in the vessel is designed with an adjustment capability in both the toroidal and poloidal directions. However, the toroidal adjustment is performed once 
during the initial installation, then welded to prevent further movement. The poloidal adjustment is controlled by the micrometer actuator which allows for positional resolution to 33 arc-seconds or better. These two adjustments provide for a precise alignment of the laser beam through the edge of the plasma. To prevent backlash in the mirror-pushrod system, a small compression spring is included in the joint assembly between the end of the push rod and the lever which moves the mirror. This spring maintains a constant pressure between the push rod, the lever, and the pivot pin. The lever connects the push rod to the axis of the pivot pins which allows the mirror to move around its horizontal axis. When the gross postional adjustments have been completed, this lever is tack welded to the end of the pivot pin to prevent any further relative movement which would upset the system alignment. At this point, the system geometry is fixed, and any further adjustment of mirror position is accomplished only with the micrometer feedthrough actuator. 


\section{BEAM AND SYSTEM ENCLOSURE EXTERNAL TO THE VESSEL}

The 20 watt $\mathrm{CO}_{2}$ laser is an invisible hazard to all tissue, particularly the eyes, and may cause significant biological damage before a person can respond to avoid the beam. The He Ne laser however, is a hazard only when viewed directly. Since the beams are not everywhere confined in light-tight enclosures, safety dictates that they be inoperable when personnel unfamiliar with these hazards are present. A safety interlock system is built into this equipment to ensure that the $\mathrm{He} \mathrm{Ne}$ laser is off, and that the $\mathrm{CO}_{2}$ laser beam is dumped to a shutter box whenever the interferometer system is not in use. The shutter box is attached to the laser output aperture and is fully capable of containing the beam in its entirety. This arrangement avoids frequent activations and deactivations of the $\mathrm{CO}_{2}$ laser discharge which could affect the stability of the output laser power.

The $4^{\prime} \times 6^{\prime}$ optical table is mounted vertically with its bottom edge about 15 inches above the floor of the DIII-D pit. The optical table is totally enclosed with protective aluminum panels on all sides, with the front of the table containing three closure panels which are removable to allow access to the optical equipment for adjustments, system troubleshooting, and analysis. Inside the enclosure, the detectors and electronics are mounted in a tightly sealed metal box which functions as an RF shield.

The beam path from the table to the mirrors, and from mirror to mirror along the beam path, is enclosed by round thin-walled aluminum tubes. To eliminate electrical conductivity along the beam tubes, each run of tube contains a G-10 fiberglass insulator ring between sections. These rings also function as connectors between sections of the tube run by bolting the tubing material directly into the fiberglass ring. The tube diameter for the outgoing beam is $150 \mathrm{~mm}$, and for the returning beam the tube diameter is $200 \mathrm{~mm}$.

Each of the external mirrors is contained in an aluminum enclosure box. These boxes are either mounted at rigid points along the beam path, or else the box is sized to just fit around the mirror itself. In the latter case, the box is bolted to the bottom support plate of the gimbal assembly and therefore moves along with the mirror during any minor adjustment of the gimbal. 
The final two mirrors on the beam path to the vessel are mounted on gimbal supports attached to the vessel. This is accomplished by using studs at specific locations on the primary closure flange. These studs are long enough to function as primary closure bolts for the flange, but are also permitted to extend above the plane of the port flange to provide bolting points for system hardware. Figure 6 shows the mounting arrangement at the 285 degree $\mathrm{R}+1$ port. As with other arrangements of hardware in close proximity to the vessel, electrically conductive loops are avoided by using G-10 fiberglass spacers and inserts to create electrical breaks at certain of the bolted connections. 


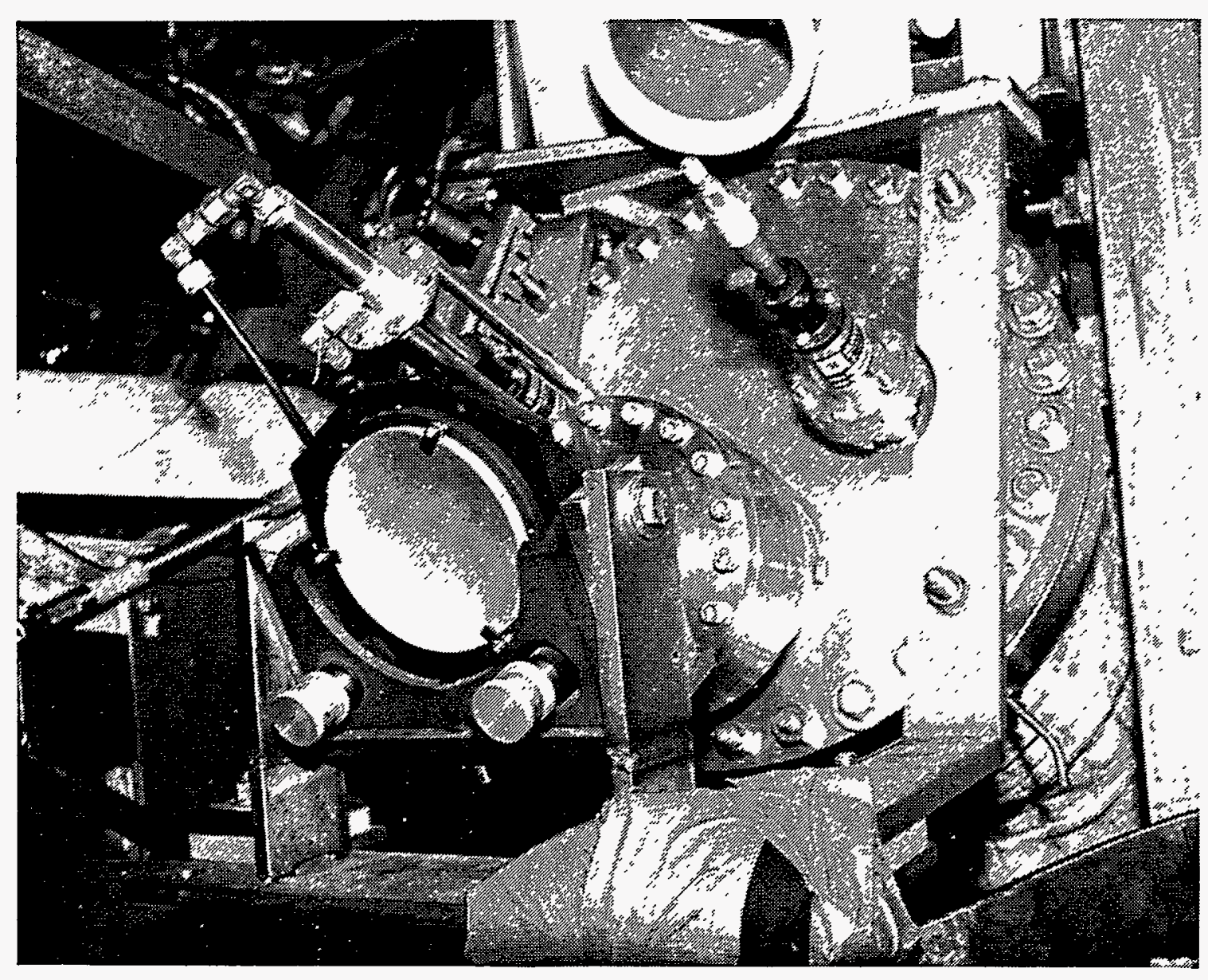

Fig. 6. External mirror mount and feed through hardware- $-\mathrm{R}+1285^{\circ}$. 


\section{IMAGING OPTICS AND DETECTION ELECTRONICS}

After the beam has traversed the plasma edge and is returned to the optical table, a second off-axis parabolic mirror focuses the beam onto a spatial filter known as a phase mirror [2]. The beam must strike the optical center of this phase mirror within an accuracy of 100 microns for the phase contrast technique to produce meaningful results. However, in the presence of mechanical vibrations which can occur during a plasma discharge, the focus can become displaced by several $\mathrm{mm}$. To cancel the effect of these vibrations, a fast, high-gain, dual-axis feedback stabilization system has been developed.

The precise position of the focus relative to the center of the phase mirror, at any time, is determined by a quadrant, liquid cooled $\mathrm{HgCdTe}$ detector. The result of this deviation measurement is instantly processed by an analog frequency compensation network whose output drives a pair of small orthogonally oriented rotatable mirrors. These mirrors are located on the laser beam return path approximately $50 \mathrm{~cm}$ in front of the phase mirror. The minor rotational movements of these mirrors exactly compensate for any system induced vibrations with the result that the returning laser beam remains focused on the precise center of the phase mirror at all times.

Two additional $\mathrm{ZnSe}$ lenses, in combination with the parabolic mirror, form an imaging system which projects an image of the vessel midplane onto a detector array. The setup is flexible and allows variable magnification to reduce the range and increase the spatial resolution, as well as providing for image orientation.

The detector is a 16-element, liquid nitrogen cooled photo voltaic $\mathrm{HgCdTe}$ linear array, followed by 16 low noise preamplifiers, operating in the bandwidth of $0.001-100 \mathrm{MHz}$. The signals are amplified, filtered, and heterodyned to a carrier frequency which can be selected between 2 and $80 \mathrm{MHz}$. Coaxial cables then transport the signals to the control room annex. This heterodyne scheme was chosen to avoid low frequency noise pickup by the 60 meter-long signal cables [3]. In the annex, the signals are down-shifted, filtered, amplified, and finally digitized at a sampling rate of two megasamples per second. 


\section{PHYSICS PARAMETERS AND CALIBRATION TECHNIQUES}

The DIII-D phase contrast interferometer measures the vertical line integrals of the plasma density fluctuations along 16 adjacent chords. These chords are located at the outer edge of the plasma at normalized radii between 0.9 and 1.15 . A $7.6 \mathrm{~cm}$ radial range is covered, with variable chord spacing set typically at $5 \mathrm{~mm}$. A bandwidth of $100 \mathrm{MHz}$ ensures that both low frequency turbulence and RF waves can be detected. Only radial wave vectors, in the wavelength range of 0.4 to $7.6 \mathrm{~cm}$, can be observed. A sensitivity of approximately $10^{9} \mathrm{~cm}^{-3}$ can be achieved with a $1 \mathrm{MHz}$ bandwidth.

The overall system is calibrated on an absolute basis using both acoustic and ultrasonic waves $(10-40 \mathrm{kHz})$ propagating in air, in a direction perpendicular to the axis of the beam path. The amplitude of these waves is measured separately by a calibrated microphone [2]. Generally, absolute calibrations are performed once per year during major DIII-D vent periods since the sound waves must be launched from inside the vessel at the midplane. However, relative calibrations are performed routinely before each plasma shot by means of high frequency sound waves generated by a small acoustical loudspeaker located in one of the mirror enclosures. 


\section{REFERENCES}

[1] R.D. Phelps, "DIII-D In-Vessel Port Cover and Shutter Assembly for the Phase Contrast Interferometer," GA-A 21532, January, 1994.

[2] S. Coda, M. Porkolab, and T.N. Carlstrom, Review of Scientific Instruments, 63, 4974 (1992).

[3] S. Coda, M. Porkolab, Review of Scientific Instruments, (1995), to be published. 